\title{
Introduction to Microwave Active Techniques and Backscatter Properties
}

\author{
Peter Hoogeboom ${ }^{1,2}$ and Ludvik Lidicky ${ }^{2}$ \\ ${ }^{1}$ Delft University of Technology, International Research Centre for Tele- \\ communications and Radar, Delft, The Netherlands \\ ${ }^{2}$ TNO Defense, Security and Safety, The Hague, The Netherlands
}

\begin{abstract}
The present article introduces active microwave techniques that are used for remote sensing of the European seas, focusing on RAdio Detection And Ranging (RADAR) and Synthetic Aperture Radar (SAR). In section 1 the ranging principle and associated topics are introduced. As an example of this technique the radar altimeter is described. Radar altimeters employ the ranging technique for one-dimensional sea surface measurements from space. In section 2, two-dimensional imaging systems will be discussed. The addition of resolving power in a second dimension perpendicular to radar ranging leads to imaging devices that exist in many varieties. The major imaging systems that are in use today for remote sensing of the sea will be described, e.g. rotating beam radars, Side looking airborne radar, Synthetic Aperture Radar and polarimetric SAR. Section 3 deals with matters concerning radar backscatter: the basic concepts and definitions, speckle, and backscatter properties of the ocean. Furthermore, Bragg scattering, angular, wind and wave dependences of the backscatter, leading to the main oceanographic applications, are discussed.
\end{abstract}

\section{Radar: Range Positioning}

This section introduces the radar as an instrument used to measure distances. First, some theoretical background and basic definitions will be established. Then practical applications will be described.

\subsection{Radar Ranging Principle}

Imagine the situation in Figure 1: a transmitter TX sends a pulse in a direction of interest. The illuminated area is limited by the directive properties of the antenna. The pulse is reflected by a distant object. The antenna cap- 
tures the signal and passes it to the receiver RX, which filters the signal and samples the echo. The time $t$ that has elapsed since transmission of the pulse can be determined from the output samples. The pulses travel at the speed of light $c$. The range $r$ to the target can be found from $t$ :

$$
r=\frac{c t}{2},
$$

where $c$ is the speed of light. The radar pulse has to travel twice the range, from radar to object and back, hence the factor 2 in the formula. In its simples form, this radar is known as a non-coherent pulse radar. It measures amplitude and time-delay. The phase of the received signal is ignored.

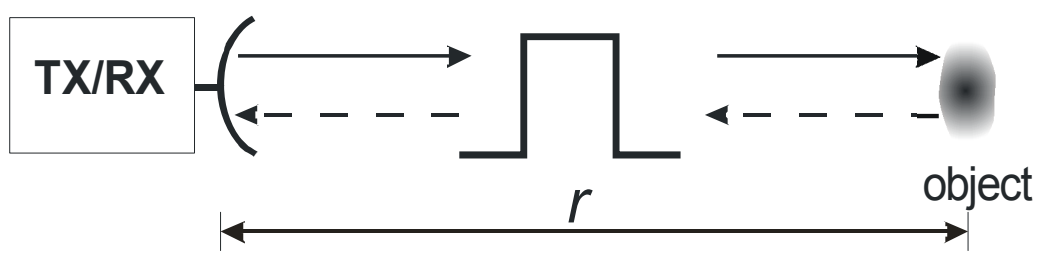

Fig. 1. Principle of the range measurement with a pulsed radar system.

\subsection{Range Ambiguities}

To monitor changes in the object or to determine its speed by measuring Doppler shift, a coherent pulse-Doppler radar is required. Modern SAR systems, scatterometers and altimeters belong to this category. Such radars measure received amplitude and phase. Successive phase measurements from successive pulses reveal the Doppler frequency shift due to motion of the reflecting object.

The coherent pulse-Doppler radar transmits pulses at a rate called Pulse Repetition Frequency (PRF). Pulse repetition is also used to increase the signal-to-noise ratio (SNR) of the radar measurement by integration. The higher the PRF, the more SNR is achieved, and the higher is the maximum Doppler shift that can be measured. But a high PRF is a potential source of range ambiguities. An ambiguity occurs when reflections from two different distances appear at the same position on the radar timeline. Consider Figure 2. The radar timeline is indicated, with several transmitted pulses. In the top drawing, echoes $\mathrm{A}$ and $\mathrm{B}$ are found in response to the first transmitted pulse. They are received before the transmission of the second pulse. 


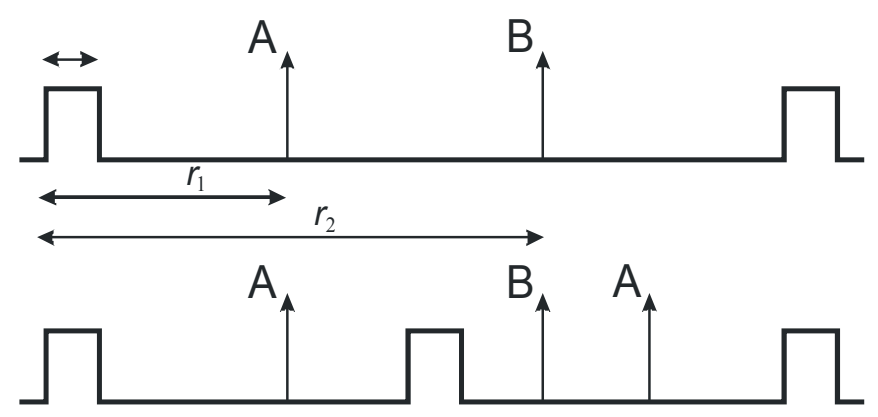

Fig. 2. Unambiguous (above) and ambiguous (below) range sampling with radar pulses.

In the lower part of Figure 2, the PRF is increased. Echo B is still from the first pulse, but the radar could erroneously interpret this echo as coming from the second pulse, hence at a shorter distance. In fact, if an echo is present at this shorter distance, it would interfere with the long distance echo $\mathrm{B}$, causing a range ambiguity. To avoid this problem, one has to ensure that all significant targets are at a distance closer than the so-called unambiguous range

$$
r=\frac{c}{2 P R F}
$$

For satellite systems, this is impossible. The distance between the satellite and the Earth is simply too large. Pulses cannot be sent at a PRF meeting the unambiguous range, because of other PRF requirements, i.e. a PRF that is high enough to sample all Doppler shifts due to the motion of the satellite unambiguously. However, by clever interpretation the unambiguous range can be compensated for the distance between the satellite and the first echoes received from the ground. As a result, the unambiguous range requirement in (2) can be relaxed. The range difference between the near and far end of the ground area that can be imaged unambiguously by the satellite, is limited by the required PRF and can be derived from (2).

\subsection{Pulse Compression and Range Resolution}

To obtain high range resolution in radar, pulses with a wide spectrum must be transmitted. This can be achieved in two ways: The first way is easy to understand. One could transmit very narrow, in theory rectangular, pulses, which represent a wide frequency spectrum. This method is applied with brute force non-coherent radar systems. Figure 3 illustrates how two equal point size objects that are close together can be separated if the pulse 
length is in the order of the distance between these objects. The dip in the received signal is just large enough to separate the two objects. This determines the radar resolution. A practical way of defining the radar resolution is through the pulse width $\tau$ of the transmitted pulse, measured at the half-power points, as shown in Figure 3 right-hand side. The pulse width $\tau$ in seconds hence determines the resolution $h$ in meters:

$$
h=\frac{c \tau}{2}
$$

For example, a pulse width of 10 nanoseconds corresponds to 1,5 meter resolution. The frequency bandwidth $B$ of this pulse is approximately equal to $1 / \tau$, or $100 \mathrm{MHz}$ in this example. Note that this definition of resolution, which is commonly used in radar, differs from the resolution definition for optical systems. The latter is often based on the visibility of line pairs at a threshold contrast level. A difference up to a factor of 2 may occur.
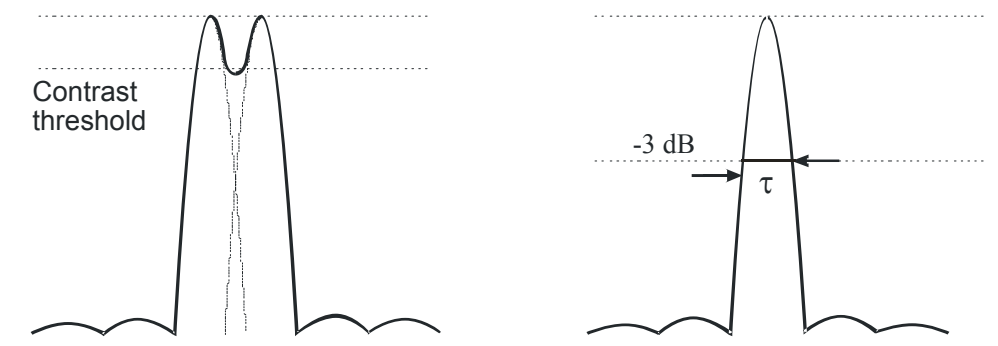

Fig. 3. Optical line pair resolution definition (left) applied to radar pulses and range resolution definition for a radar system (right).

The second way to achieve high resolution is to transmit longer pulses haped in such a way that they will have a broad bandwidth. The involved manipulation of the carrier wave implies the use of a coherent pulseDoppler radar. An example of a common pulse shape used in radar is a chirp. Roughly speaking, as long as the bandwidth of the narrow pulse in the first example and the long, chirped pulse are the same, transmitted information (and thus the system resolution) will be the same.

To retrieve the resolution from the long, chirped pulse responses, an operation called matched filtering is needed. A matched filter performs a correlation of a received signal with a replica of the transmitted waveform, hence the term "matching". The matched filter output shows narrow sharp peaks at centre positions of the original pulses, see Figure 4. Since a matched filter transforms long pulses into short ones, this operation is also called pulse compression. Pulse compression is often used in coherent radar systems for power budget reasons. The average output power of the ra- 
dar is important in achieving sufficient SNR. If a radar transmits shorter pulses to obtain finer geometrical resolution, the peak output power must be increased to maintain the same SNR. Due to technical limitations, a longer pulse of lower peak power is often preferred.

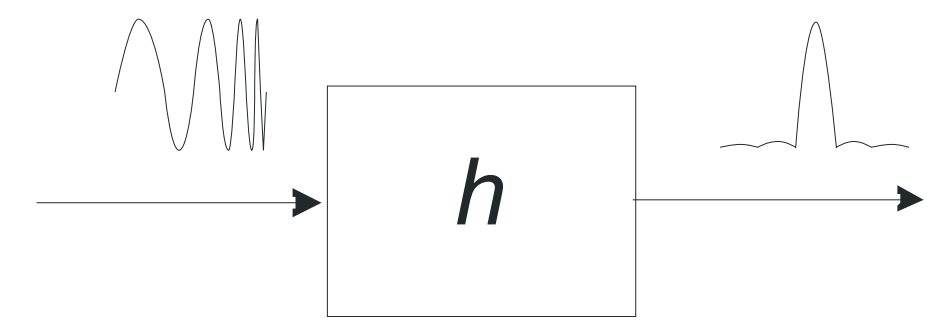

Fig. 4. Pulse compression with a matched filter (h); a long chirp (left) is converted to a narrow, high energy pulse (right).

A theory dealing with design of chirped pulses is called waveform design (Levanon 2004). Also, the pulse compression procedure influences system resolution. Mostly, the Maximum Likelihood Estimation (MLE) is used to perform the pulse compression. The resolution $\mathrm{h}$ obtained is given by equation 17.3, where again the pulse length $\tau$ is $1 / \mathrm{B}$, the bandwidth of the pulse. As an alternative, Minimum Mean Square Error Estimation (MMSEE) can be used to enhance the resolution. However, MLE is easier to perform and it is more robust in the presence of noise (Haykin 2006).

\subsection{Applications}

A typical application of a ranging device in the remote sensing of seas is the altimeter. Its basic setup is provided in Figure 5. It is a down-looking coherent pulse-Doppler radar mounted on a satellite platform. The radar has a pencil-beam antenna which is pointed perpendicular to the Earth surface. The use of a narrow pencil beam enables application of the altimeter over oceans and seas, even close to the coast. This is of importance for applications in the European seas.

The radar transmits chirped pulses to obtain a high range resolution and sufficient SNR. Since sea water constitutes a very good reflector, the sea surface behaves as a mirror reflecting radar pulses back to the satellite. The incoming pulses are compressed as shown in Figure 4. Each range measurement provides an actual height of the instrument above the sea surface. If the position and distance of the satellite to the centre of the Earth are known, the measurement represents a sample of the water level at the Earth's surface. Calibration and correction for atmospheric delays are 
needed to bring the results to a level that is of interest to the user community. Nowadays, the accuracy of the measurements after correction and calibration is at millimetre level.

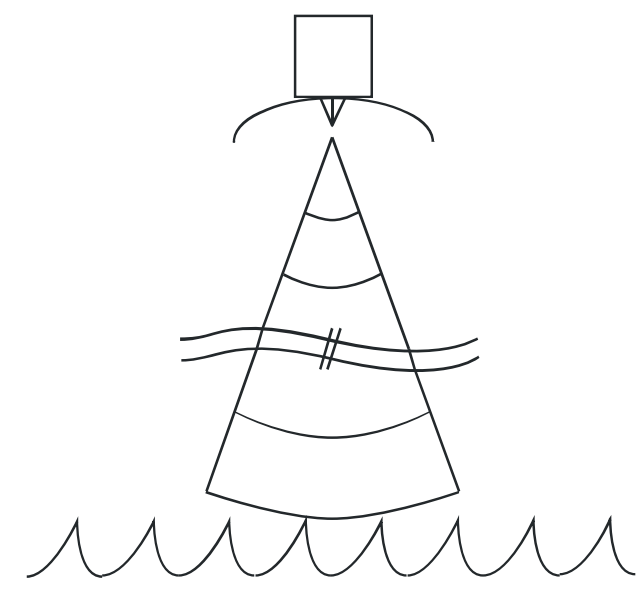

Fig. 5. Space borne radar altimeter measurement concept.

As the satellite orbits around the Earth, a complete 3-dimensional picture of the water level is created. In addition to the general height measurement, the altimeter provides average waveheight and windspeed. Waveheight is deduced from the shape of the reflected pulse, whereas the wind speed can be found from the backscatter level. Although more information can be retrieved, most often, altimeters are used to determine wave height and wind speed in addition to the primary height measurement.

\section{Azimuth Positioning, Synthetic Aperture Radars}

This section will take radar instruments from one dimensional ranging to the next level: two-dimensional radar sensing and signal processing in range and azimuth will be introduced. Here, azimuth means a direction perpendicular to the range direction, parallel to the antenna motion direction. Historically, the non-coherent radar systems used for range and azimuth positioning evolved from one-dimensional systems described above. By moving or rotating the antenna, adjacent azimuth locations are imaged in addition to the ranging. Finally, the requirement for higher resolution has led to the development of Synthetic Aperture Radar (SAR). A more complex coherent pulse-Doppler radar system is required for this application and also extensive digital processing capability is needed. 


\subsection{Rotating Beam Radars}

Rotating beam radars are traditionally non-coherent radars that use a rotating antenna with a narrow beam width in the direction of rotation (azimuth). The image is formed by plotting the range measurements for each azimuth angle at the correct position in a 2-dimensional display. If the systems are used at long ranges, the azimuth resolution, which degrades with range, becomes noticeably crude.For a long time, rotating beam radars have been mainly used for (maritime) traffic applications, e.g. as ship radars to monitor the presence and movements of other ships. They are also used as coastal radars and as harbour radars and at airports to monitor the traffic situation. Nowadays rotating beam radars are also in use for remote sensing of the seas. Wave fields can be imaged by these radars and they can visualise surface slicks from oil spills etc. The radar itself being stationary, delivers an image at every rotation of the antenna. The resulting pile of $2 \mathrm{D}$ wave images can be analysed in a computer to provide wave spectra, water current and water depth information.

\subsection{Side-Looking Airborne Radar (SLAR)}

From a technical point of view SLARs strongly resemble the rotating beam radar. The antenna rotation is replaced by an antenna translation, by mounting a non-coherent radar on an aircraft in such a way that it looks to the side(s) of the aircraft. Again, a 2D image is created. SLAR systems are in use throughout Europe for monitoring coastal areas and seas. They are capable of detecting the positions of ships and can detect oil spills. In these applications the crude azimuth resolution at longer ranges is not a major drawback. The relatively low system cost and ease of data processing, when compared to SAR systems, make them a favourable choice.

\subsection{Principle of SAR}

The major drawback of SLAR systems, i.e. the crude azimuth resolution, is overcome by the SAR principle. In radar systems, the azimuth resolution is determined by the antenna footprint, which increases with range. To maintain sufficient resolution at long range, one could increase the antenna size. The larger the antenna, the smaller the antenna footprint, the finer the resolution. However, a fine resolution at a long range will place unrealistic requirements on the antenna diameter. Consider a satellite carrying a radar. Many satellites orbit at heights of about $800 \mathrm{~km}$. At such a radar range, the 
antenna footprint will cover in azimuth several kilometres. To image ocean features, the azimuth resolution of such a system is unacceptable.

In order to circumvent this problem, the idea to synthesise a radar antenna aperture was suggested; hence the term synthetic aperture radar. The principle of a SAR system is the following: a moving, coherent pulseDoppler radar takes range measurements along the path of its motion. These measurements are coherently processed to obtain a 2D image in range and azimuth direction. Since the azimuth measurements along the path are coherently combined, the same azimuth resolution is obtained as if a large antenna was used.

Another way of understanding the principle of SAR is to consider the change in Doppler frequency as the antenna moves along the objects to be imaged. For each object that appears in the beam, a positive Doppler shift is recorded as the radar approaches the object. In the middle of the beam the Doppler shift is zero, as the radar passes by. Finally, the Doppler shift is negative as the radar moves away from the object, until it is no longer within the scope of the radar beam. The radar has thus recorded a Doppler frequency chirp for each object. Through the use of the pulse compression technique previously described, this long chirp can be compressed to a narrow pulse.

Consider Figure 6. Suppose the radar moves in the azimuth direction and looks in the range direction at four point targets depicted on the right. The range positions of incoming pulses for each azimuth position are depicted on the left. The response for each object extends in range due to the long chirped pulses that are transmitted and in azimuth due to the long history that is recorded by the antenna. The goal of SAR processing is to obtain the focused points on the right from the data on the left. This task is achieved by means of 2D matched filtering. Observe that the shape of the signatures is range dependent. This complicates the processing. Various methods have been proposed (Carrara 1995, Soumekh 1999).
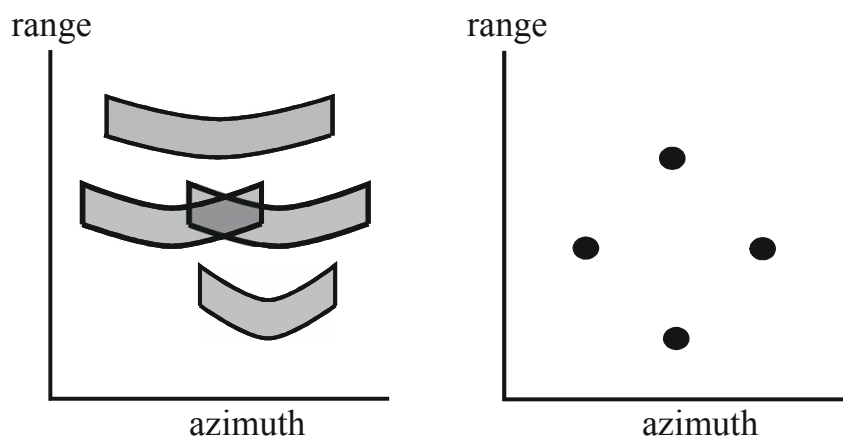

Fig. 6. Two-dimensional SAR signal responses and their processing. 
The width of the signatures in azimuth depends on the length of the antenna: the longer the antenna, the narrower the beam, and the narrower the signature. This means fewer points to process, but also a reduced resolution. The maximum azimuth resolution that can be obtained with a side looking SAR system is approximately half the antenna length in azimuth direction. Apparently, with SAR there is no dependence of resolution on range or wavelength, which makes it a powerful imaging technique.

\subsection{Azimuth Ambiguities and Moving Objects in SAR}

Azimuth ambiguities occur if the scatter in an image pixel is not uniquely related to one azimuth position. They are caused by strong reflections entering through the side-lobes of an antenna. Furthermore, moving objects are displaced from their actual position in the SAR image, which also can be considered as an ambiguity.

The SAR system is designed in such a way that stationary targets illuminated by its antenna's main lobe will be imaged unambiguously. Since PRF plays the role of a sampling frequency in the azimuth direction, one makes sure that this spatial sampling frequency is in a proper relation to the spatial extent of an illuminated area (radar footprint), i.e. that the Nyquist sampling criterion is satisfied. If a strong reflection enters through a side-lobe, it falls outside the intended spatial extent. This violates the Nyquist criterion and leads to aliasing. Normally, squinted targets are too weak to manifest themselves in the processed image because they are suppressed by the gain difference between the main lobe and side-lobes. Strong scatterers, however, are sometimes visible. They appear as misplaced objects or 'ghosts'.

Objects having a speed component in the direction of the radar are Doppler shifted. This Doppler shift leads to a squinted projection of the object in the SAR image. The squint angle is proportional to the object's speed. If its speed is low, the object is properly sampled and it mostly appears misplaced in the azimuth direction. Typical examples are ships separated from their wakes (see Figure 7).This example is taken from the first ESA ERS-1 image ever released, which was acquired on 27 July 1991. It shows an area of the North Sea, north of the Frisian island Terschelling in The Netherlands, with several ships and their wakes. The ships are slightly displaced from the wakes due to their speed. Note that in calm conditions the ship wake can be seen over distances of $20 \mathrm{~km}$ and more.

If the speed of an object is high, it will not be properly sampled by the radar. This is comparable to the previously discussed situation of the 
squinted object that entered through a side-lobe. In this case, the object will be smeared and is no longer visible in the SAR image.

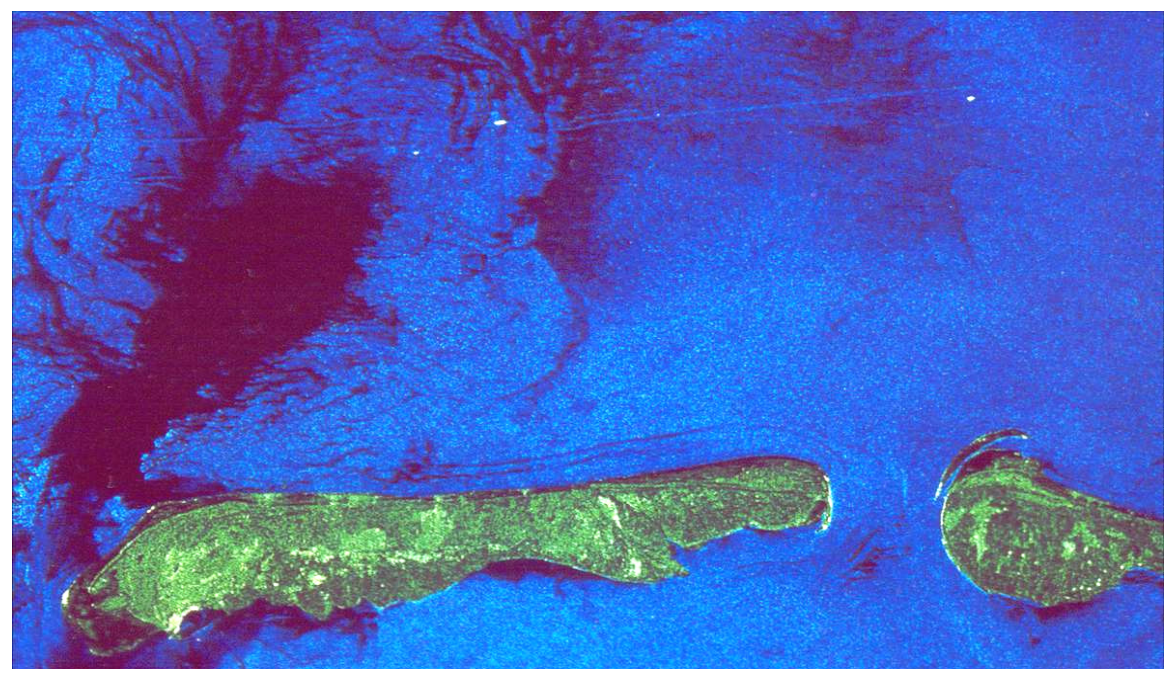

Fig. 7. First (false colour) ERS-1 image of ship wakes in the North Sea near Terschelling, The Netherlands. C ESA 1991.

\subsection{Polarimetric SAR}

The single quantity measured by a radar is the strength of the backscatter. In remote sensing this quantity is used in many ways to obtain quantitative information from the sea. Sometimes more information about scattering mechanisms, shape and structure can be obtained by combining measurements from various polarisations. This can be achieved by a polarimetric SAR. Such a radar has two receiving antennas, which receive the vertical and the horizontal polarisation, respectively.

The transmitter alternatively sends out vertically and horizontally polarised waves. Consequently, all four polarisations $(\mathrm{HH}, \mathrm{HV}, \mathrm{VH}, \mathrm{VV})$ and their phase differences are measured and recorded in a scattering matrix. This matrix is the starting point for polarimetric analysis. Note that the information content of a polarimetric radar is four times the amount of a normal radar. Hence, the data stream is a challenge.

The polarimetric technology is nowadays also available on some satellite radar systems. The measurement of the cross polarised components is a challenging task, as the signal level is approximately a factor 10 smaller than the co-polarised. For this reason and also to limit the amount of data, 
some polarimetric modes are limited to a subset of the scattering matrix, for example $\mathrm{HH}, \mathrm{VV}$ and their phase difference, or one cross polarisation (in theory $\mathrm{HV}$ equals $\mathrm{VH}$ ) and one co-polarisation.

\section{Radar Backscatter}

Microwave reflections are different from optical reflections, which the human eye is experienced in interpreting. Radar backscatter is often presented as an image and may at first glance resemble an optical picture, but it is not. If the interpreter does not realise this, the interpretation may be erroneous. In understanding microwave reflections, the wavelength should be taken into account. The relative size of the scatter cells (shapes and structures, relative to the wavelength) on an object determines the reflection behaviour, apart from conductive and dielectric properties. A scratched mirror is useless for reflecting an optical image. However, radio waves with a wavelength 1000 times longer will not be influenced by the scratches and will be reflected as if the mirror was perfect. Figure 8 illustrates several surface scattering situations, from forward scattering, occurring on smooth surfaces, to omni-directional scattering on rough surfaces.
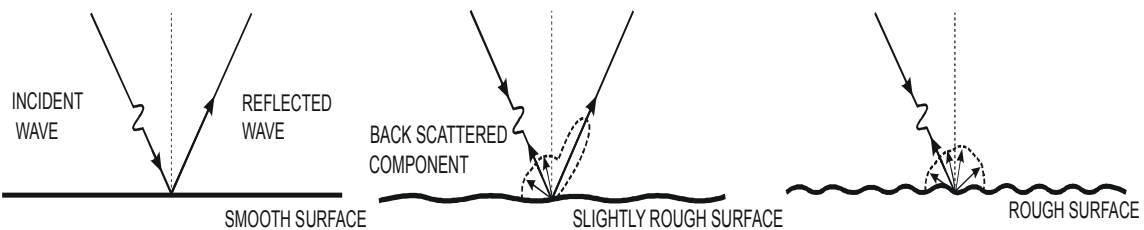

Fig. 8. Surface scattering mechanisms for smooth, slightly rough and rough surfaces.

\subsection{Scattering Definitions}

Microwave scattering from objects may occur in various forms. The properties of the object determine what type of reflection occurs. A distinction can be made between single point target reflection, multiple-points reflection, also known as extended or surface scattering, and volume scattering. In discussing sea surfaces, the dominant scattering type is surface scattering, as the electromagnetic wave does not penetrate into the water. Ships, buoys and other objects in the sea will contribute target reflections consisting of one or more point targets. Depending on wavelength, sea ice can be penetrated by microwaves. In this case, not only surface scattering is im- 
portant, but also the volume scattering from the volume that is illuminated by the radar radiation.

\subsubsection{Radar Cross-Section}

Consider the well known radar equation

$$
P_{\text {rec }}=P_{t r} \frac{G^{2} \lambda^{2}}{(4 \pi)^{3} R^{4}} \sigma
$$

where $P_{r e c}$ is received power, $P_{t r}$ transmitted power, $G$ the antenna gain, and $\lambda$ the radar wavelength. $\sigma$ stands for the radar cross-section. It is measured in square meters. The radar cross-section of an object can be thought of as the area of a perfectly conducting sphere that would give rise to the same level of reflection as observed from the object. Radars for remote sensing applications are usually calibrated in such a way that their outputs are radar cross-section numbers. For reasons of large dynamic range, the numbers are often given in decibels. This logarithmic compression reduces the dynamic range and enables easy calculations.

\subsubsection{Sigma Naught for Surface Scattering}

A reflecting surface can be thought of as a collection of point targets.

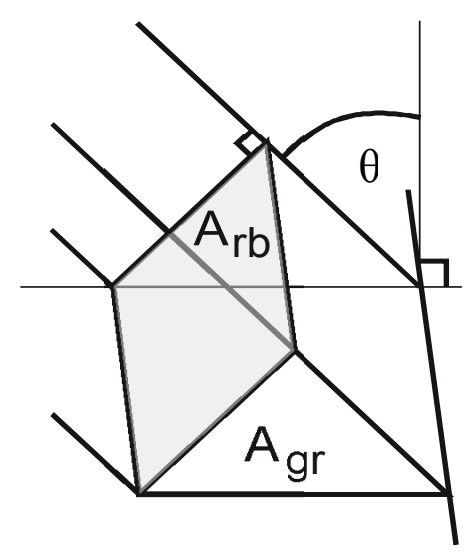

Fig. 9. Definition for ground referenced and radar beam referenced radar backscatter coefficients.

The radar backscatter per unit area of this surface is known as sigma naught or $\sigma_{0}$. It is a dimensionless quantity, indicating the radar crosssection in square meters per square meter earth surface. This results in 


$$
\sigma_{0}=\frac{\sigma}{A_{g r}}
$$

where $A_{g r}$ is the resolution cell projected on the ground (Figure 9). $\sigma_{0}$ is mostly used with satellite systems. For sea skimming systems like ship radars another definition is sometimes used, based on the cross section of the radar beam $A_{\mathrm{rb}}$, rather than the illuminated ground area $\mathrm{A}_{\mathrm{gr}}$. The backscatter coefficient $\gamma$ can be easily related to $\sigma_{0}$ :

$$
\gamma=\frac{\sigma_{0}}{\cos \theta}
$$

\subsection{Speckle}

Objects that have a larger size than the resolution of the radar will deliver several smaller reflections to the radar, which are then combined in the radar system into one reflection. Such reflections will possess special properties. The individual reflections are combined in amplitude and phase, known as coherent addition.

Two reflections with equal amplitudes, but opposite phase, will cancel each other out. If the reflections are in phase, the amplitude will double. Phase changes are largely due to small distance variations between the scattering object and the radar antenna. A moving sea surface gives rise to many phase changes. The radar reflection will show a continuously changing behaviour in amplitude and phase. This effect is known as speckle or clutter and should be separated from the mean radar backscatter value.

\subsection{Sea Backscatter}

Several mechanisms contribute to the radar backscatter of the sea. The main effect is Bragg reflection. The backscatter level is influenced by wind, waves, currents and surface slicks, like oil pollutions or natural slicks. Other important effects are the angular dependence of radar backscatter, the dependence on wind speed and wind direction, and on polarisation. Finally, the moving sea surface decorrelates rapidly. Any coherent radar measurement process like SAR image formation will be hampered by the decorrelation. Nevertheless, many interesting features can be observed in the seas, especially in the European seas. These seas possess many delta areas with strong tidal currents and areas with layered structures of saline and fresh waters or water masses of different temperatures. 


\subsubsection{Bragg Scattering}

Bragg scattering is a resonant scattering mechanism, originally discovered and described by Bragg in 1913 for X-ray reflections from molecular grates in solid materials. The same mechanism is responsible for radar reflections on wind-generated short gravity-capillary waves. These waves can have wavelengths in the $\mathrm{mm}$ to $\mathrm{dm}$ range and increase to longer waves as a function of the fetch of the wind over the surface. The reflections are combined in the radar antenna. At this point, a coherent (amplitude and phase) addition of the reflections is performed. Those reflections that are perfectly in phase will lead to a high amplitude. All other reflections are cancelled out Figure 10 depicts the geometry.

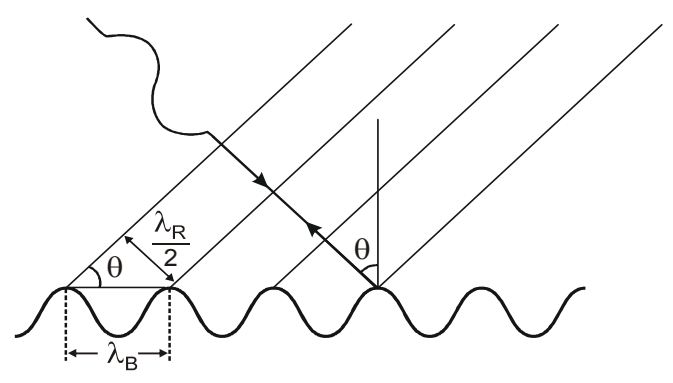

Fig. 10. Bragg resonant scattering on sea waves.

Electromagnetic waves with a wavelength $\lambda_{R}$ fall onto the surface at an incidence angle $\theta$. Capillary waves with a wavelength $\lambda_{\mathrm{B}}$ fulfil the resonance condition if

$$
\frac{\lambda_{R}}{2}=\lambda_{B} \sin \theta
$$

Note that the radar wave will travel to the water surface and back, doubling the travelled distance, hence the factor 2 . Wind is needed to image the ocean surface, because without the short gravity-capillary waves the surface will not be visible in the radar image.

\subsubsection{Wind Dependence}

The radar backscatter level depends on the wind speed, because the spectral density for the Bragg resonant wave is related to the wind speed and the radar backscatter in turn is related to this spectral density. Consider a case where the radar is looking upwind and at a constant incidence angle. In this situation, a nearly perfect linear dependence is found between the radar reflectivity in $\mathrm{dB}$ (logarithmic scale) and the logarithm of the wind 
speed. The absolute value of the reflectivity depends on radar parameters, like polarisation and radar frequency, and on the viewing geometry.

\subsubsection{Incidence Angle Dependence}

The generalized radar backscatter dependence on incidence angle for moderate wind speeds is shown in Figure 11. The theoretical Bragg curves are shown. For extreme incidence angles (near nadir and near grazing), the Bragg resonant scattering mechanism is dominated by other effects.

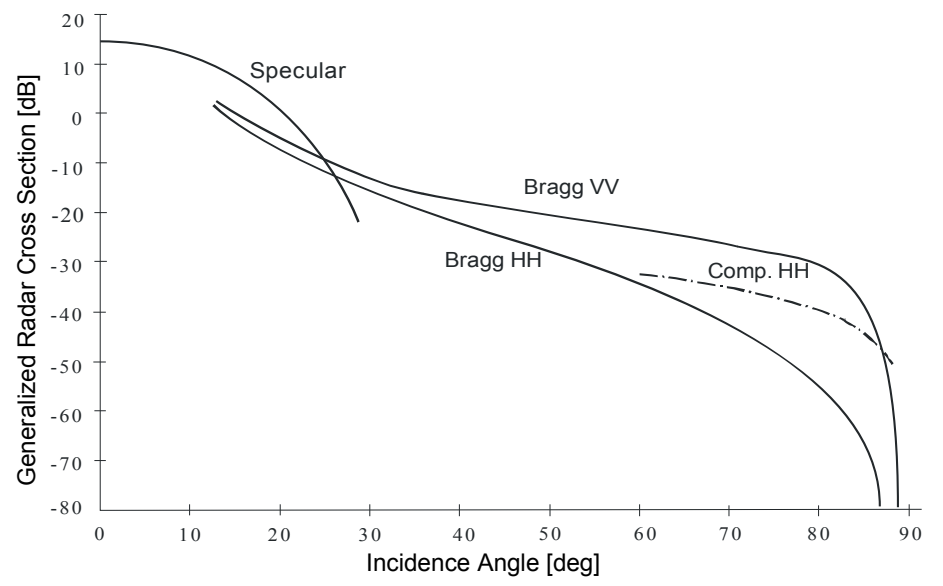

Fig. 11. Radar backscatter versus incidence angle for moderate wind speeds.

Specular reflections occur from $0^{\circ}$ (nadir reflection) to $30^{\circ}$. The upper value is in line with the maximum wave steepness of $60^{\circ}$. This stems from the fact that normal sea waves, fulfilling Stokes laws, exhibit a minimum crest angle of $120^{\circ}$. If this value is exceeded, the wave will break, which leads to strong spikes in the radar signal at large incidence angles, i.e. $80^{\circ}$ to $90^{\circ}$ (near grazing). In this angular region, multipath effects and shadowing occur as well. The average backscatter coefficient strongly reduces when the incidence angle approaches $90^{\circ}$ or grazing.

\subsubsection{Polarisation Dependence}

The conductive and dielectric properties of seawater lead to a distinct behaviour of the radar backscatter for various polarisations. The polarisation of an electromagnetic wave (Horizontal or Vertical) indicates the plane of the electric field of the wave.nIn the specular region $\left(0^{\circ}-30^{\circ}\right)$, there is no noticeable difference between horizontal and vertical radar wave polarisation. In the Bragg dominated region $\left(30^{\circ}-70^{\circ}\right)$, the vertical polarisation 
yields a stronger return signal. However, sea spikes and breaking waves generate strong returns at horizontal polarisation. At larger angles, above $60^{\circ}$, these effects dominate the Bragg scattering as indicated by the dashed line in Figure 12. This line is based on composite models. At extreme angles near grazing, a cross-over occurs and the horizontal polarised signal is stronger than the vertical polarised Bragg scatter.

\subsubsection{Decorrelation}

When the radar return signal from a single resolution cell is monitored over some time, one may observe random variations in amplitude and phase, leading to incoherency of the time series. This decorrelation effect is of particular importance for SAR systems, as they achieve a high resolution by combining measurements from various antenna positions along a straight track during the SAR aperture time. If this aperture time exceeds the decorrelation time, the SAR image may not achieve the expected resolution in azimuth (antenna flight) direction. In radar range direction, the resolution is not altered. As a consequence, waves are sometimes not well imaged. They can be slightly rotated, broken up or blurred. Typical decorrelation times for the sea surface are $10-100$ millisecond, depending on sea state, radar frequency and incidence angle. SAR aperture times vary with systems, resolution and observation distance. Typical values are 50 milliseconds to 1 second for satellite SAR systems.

\subsection{Wind Vector Determination}

Radar can be used to determine the wind vector over open seas, i.e. away from the coast. There are various methods, based on SAR, radar altimetry and wind-scatterometry. The latter is the usually applied and most accurate method. It is based on the measurement of wind speed and wind direction using a multi-beam radar instrument.

The radar backscatter level not only depends on wind speed, but also on wind direction with respect to the radar look direction. Figure 12 shows the general azimuth dependence. At upwind (the radar looks into the wind), the highest backscatter occurs. Downwind values are also high, but slightly lower than the upwind value. At crosswind positions, minima occur in the curve. A 3 beam scatterometer, as used by ESA on their satellites, with a $45^{\circ}$ azimuth angle separation between the beams, is a good solution to determine the wind vector, even though an $180^{\circ}$ ambiguity may occur, e.g. between the 2 crosswind positions in Figure 12. Such ambiguities can be resolved from a regional wind field analysis. 


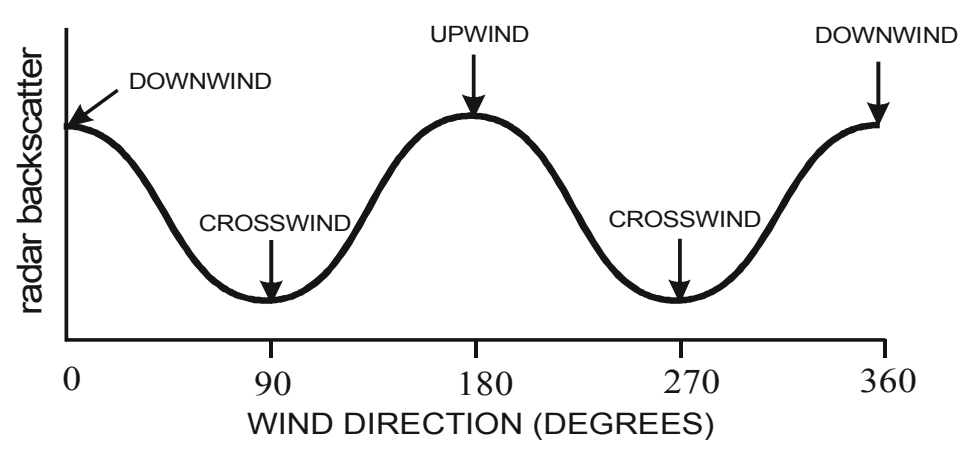

Fig. 12. Azimuth dependence of radar backscatter for the sea surface.

\subsection{Modulation Processes}

The wind generated Bragg waves are easily influenced by external factors like sea waves, bottom topography, slicks and precipitation. This modulation leads to their visibility in SAR images. The ocean wave imaging and bottom topography visualisation will be discussed.

\subsubsection{Long Gravity Waves}

Long gravity sea waves introduce several modulation effects in the radar backscatter, leading to a generally good visibility of such waves in radar images. The main effects are slope modulation and hydro-dynamical modulation. Slope modulation is caused by the change of the local incidence angle of the radar wave due to the local ocean wave inclination. The effect depends on the average incidence angle. This modulation effect has a strength in the order of a few $\mathrm{dB}$.

Hydro-dynamical modulation is caused by the motion of the surface due to the passing sea waves. This motion will lead to a stretching and straining of capillary wave fields, thereby modifying their amplitudes and thus the radar backscatter. Furthermore, this motion of the surface may lead to Doppler velocity shifts in the radar signal, which can lead to a non-linear mapping of the sea wave in the radar image. The effect can be compared to the displacements of ships from their wake due to their velocity.

\subsubsection{Bottom Features}

Coastal seas with limited depth and delta areas can express bottom features under certain conditions in the radar image, even though the radar wave does not penetrate into the water. These effects were first discovered in 
SLAR images over the North Sea by De Loor (De Loor and Brunsveld van Hulten 1978). The mechanism of bottom topography imaging is explained in Figure 13.

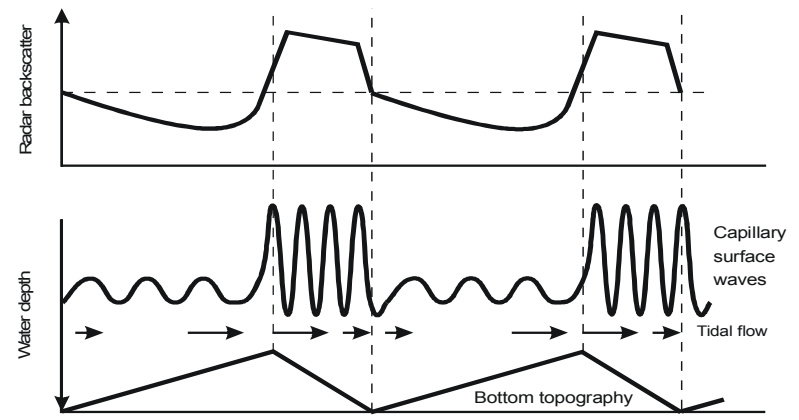

Fig. 13. Sea bottom topography imaging mechanism.

Consider a sea surface where the wind is generating capillary waves. A tidal water current moves a water body over the undulated shallow water (depth less than approx 25 meter). Water particles will accelerate over the underwater dunes and decelerate over the valleys to maintain a constant total water flow. On the surface, these changes in local water current will modulate the capillary waves. During accelerations they are stretched, lowering their amplitude. This causes a reduced radar backscatter. When decelerating, the capillary waves are compressed, thus intensifying the radar backscatter. As the disturbed capillary wave spectrum quickly decays to the equilibrium spectrum, the effect in the radar image also quickly disappears as indicated in the upper part of Figure 13.

\section{References \& Bibliography}

Carrara WG, Goodman RS, Majewski RM (1995) Spotlight SAR: Signal processing algorithms. Artech House

De Loor GP, Brunsveld Van Hutten HW (1978) Microwave measurements over the North Sea. Bound-layer Met 13: 119-131

Levanon N, Mozeson E (2004) Radar Signals. Wiley \& Sons

Haykin S (2006) Adaptive radar signal processing. Wiley \& Sons

Henderson FM, Lewis AJ (1998) Principles and applications of imaging radar. Wiley \& Sons

Skolnik MI (2001) Introduction to radar systems. McGraw-Hill

Soumekh M (1999) Synthetic aperture radar signal processing. Wiley \& Sons

Ulaby FT, Moore RK, Fung AK (1981-1986) Microwave remote sensing. vol. I, vol II, Addison-Wesley, vol. III, Artech House 